\title{
Kernos
}

Revue internationale et pluridisciplinaire de religion grecque antique

19 | 2006

Varia

\section{Présentation du dictionnaire de la mythologie grecque on line}

\section{Ezio Pellizer}

\section{OpenEdition \\ Journals}

Édition électronique

URL : https://journals.openedition.org/kernos/456

DOI : 10.4000/kernos.456

ISSN : 2034-7871

\section{Éditeur}

Centre international d'étude de la religion grecque antique

Édition imprimée

Date de publication : 1 janvier 2006

Pagination : 245-248

ISSN : 0776-3824

\section{Référence électronique}

Ezio Pellizer, «Présentation du dictionnaire de la mythologie grecque on line », Kernos [En ligne], 19 2006, mis en ligne le 24 mai 2011, consulté le 24 août 2022. URL : http://journals.openedition.org/ kernos/456 ; DOI : https://doi.org/10.4000/kernos.456 


\title{
Présentation du dictionnaire de la mythologie grecque on line
}

\author{
par Ezio PELLIZER
}

Le Groupe de Recherche sur le Mythe et la Mythographie de l'Université de Trieste (GRIMM) a rassemblé un abondant matériau pour un projet de Dictionnaire Etymologique de la Mythologie Grecque. La recherche, d'abord menée dans le cadre d'un mémoire de licence (de Carla Zufferli), est conçue comme une mise à jour du dictionnaire Carnoy². Désormais dépassé, ce dernier ouvrage se montrait trop enclin à chercher des étymologies « pélasgiques », avec pour effet récurrent d'«éclairer » obscura per obscuriora. Loin de nous l'intention de remplacer les nombreux Dictionnaires de Mythologie existant par ailleurs : aussi le contenu des articles se limite-t-il à fournir quelques informations essentielles sur le personnage et sur les principales sources qui en parlent. En revanche, les articles examinent ensuite avec soin les étymologies les plus plausibles des noms de maints personnages du mythe grec. La méthode de travail se fonde sur un examen attentif des étymologies proposées par les spécialistes, depuis le vieux répertoire de Pape et Benseler jusqu'aux hypothèses les plus récentes (Wathelet, von Kamptz, Zamboni, van Windekens, Salvatore, etc.) : on s'efforce de choisir, là où c'est possible, parmi les propositions les plus vraisemblables et les mieux fondées du point de vue linguistique.

Il nous a également semblé que rendre accessible ce matériau on line sur le réseau Internet revenait à fournir un instrument de recherche très souple. capable d'aider, par exemple, à comprendre le système de signification des noms employés dans le discours mythique. En effet, souvent dans le mécanisme de dénomination "rigide » que constitue l'anthroponyme, se cache un énoncé qui intéresse, par exemple, les attentes des parents et des grandsparents à l'égard de leur descendance; il s'agit donc d'explorer l'anthroponyme en sa qualité de micro-énoncé narratif ${ }^{3}$.

Dans le système des anthroponymes les plus anciens, à côté des noms à fonction qualificative ou descriptive visant le personnage même, on trouve

\footnotetext{
${ }^{1}$ Adresse web: http://www.univ.trieste.it/ grmito/

2 A. CARNOY, Dictionnaire étymologique de la mythologie gréco-romaine, Louvain 1957; $c f$. aussi, pour le public anglophone, A. RoOM, Room's Classical Dictionary. The Origins of the Names of Characters in Classical Mythology, London, Boston, Melbourne and Henley 1983.

${ }^{3} \mathrm{Cl}$. CALAME, «Récit et anthroponymes : noms de laconiennes en figures de rhétorique », in id., Le récit en Grèce ancienne. Enonciations et répresentations de poètes, Paris 1986, (trad. it. Roma / Bari, Laterza 1988), p. 153-161 (chap. VII).
} 
aussi des références aux vertus et qualités dynastiques (celles du père ou du grand-père); s'y implante ainsi un système qu'on pourrait qualifier de " patrocélébratif », par le truchement duquel l'enfant reçoit un nom qui évoque les qualités manifestées ou les entreprises accomplies par son géniteur ${ }^{4}$.

La liste des exemples aurait vite fait de s'allonger: Pisistrate, Eurysace, Gorgophonos, Télémaque, etc. Un cas au moins avait déjà été remarqué par les Anciens : le nom de Néoptolème, donné à Pyrrhos parce que son père Achille avait commencé à combattre lorsqu'il était encore tout jeune (Pausanias, $\mathrm{X}, 26,4)$. On pense aussi au nom de Cléopâtre, «la gloire de son père ».

Mais ce dictionnaire en ligne peut aussi aider à résoudre ou du moins à débrouiller l'interprétation de noms féminins tels qu'Antigone, qui ne signifie pas «against the birth», ou pire ancore « against the womb»; ou encore ceux de Déjanire, d'Andromaque, de Déidamie, d'Antianeira ou de Lysistrata, qui n'expriment pas d'obscurs fantasmes de jeunes filles terrifiantes, de femmes homicides capables d'insuffler la terreur, d'anéantir le mâle (anèr), mais tout simplement la tentative de décrire, dans le nom de la petite fille, les talents guerriers, authentiques ou présumés, de leur noble père. Plus précisément, il semble qu'une réflexion générale sur l'anthroponymie (masculine et féminine) permettrait d'éviter que se répandent des étymologies fantaisistes et invérifiables (par ex. expliquer Edipe par oida- et poùs, «celui qui connait le pied $» !)$.

Il convient de mentionner le cas où se manifeste avec le plus de conviction le lien entre l'anthroponyme et les aventures vécues ou les entreprises accomplies par le héros déjà adulte. Là, à moins d'attribuer à l'acte d'imposition du nom de véritables vertus prophétiques «nomen est omen», la question est de savoir si le nom lui-même ne véhicule pas un indice énonciatif de fictionnalité. Si tel personnage, comme Polynice (Polyneikes), porte un nom qui signifie «aux nombreuses querelles", et s'il doit justement sa célébrité aux disputes au cours desquelles il a affronté son père et s'est battu à mort avec son frère, on se prendra aisément à penser que son nom a dû jaillir à l'esprit du narrateur de l'histoire a posteriori, qu'il a été «trouvé », «inventé » en fonction de la fiction. En bonne logique, le cognomen ex virtute devrait être attribué après que le sujet a manifesté ses qualités, et non dès sa naissance.

D'autres aspects tels que la «caconymie» (Kopreus, Aischros, Pentheus) ou bien les vicissitudes intéressantes de noms qui rendent mémorables des mésaventures ou malheurs dynastiques, comme Megapenthes, Odysseus (dans l'explication qu'en donnent les anciens rhapsodes homériques eux-mêmes), ou encore les anthroponymes qui renvoient à des animaux (serait-ce des «totems » ?), méritent réflexion : le cheval, plus rarement la génisse, en Grèce

\footnotetext{
${ }^{4}$ Voir surtout l'important article de M. SULZBERGER, «ONOMA FПONYMON. Les noms propres chez Homère et dans la mythologie grecque », REG 39 (1926), p. 381-447.

${ }^{5}$ Cf. le beau travail de Nicole LoRAuX consacré à «Polyneikes eponumos : les noms des fils d'CEdipe, entre épopée et tragédie », in Cl. CaLAme (éd.), Métamorphoses du mythe en Grèce antique, Genève, 1988, p. 151-166.
} 
surtout, hantent Leucippos (Leucippe), Cyanippe, Hippolyte à côté de Periboia, Alphesiboia; et que dire de Autolycos ou Autoleon?

Le travail est actuellement en cours de rédaction en langue italienne et de révision scientifique. Il est élaboré sous forme de banque de données pour une présentation WEB sur le serveur de l'Université de Trieste, assurée par le Laboratoire HiReMa (Historical Resources Management) LAB dirigé par Nevio Zorzetti. On peut en consulter dejà un vaste échantillon démonstratif de quelques lettres (A, B, C, D, E, F, G, I et R, S, T, U, Z), avec un index des entrées par ordre alphabétique, qui compte (au début de 2006), 500 entrées environ. Plus ou moins 1000 entrées sont prévues, avec la possibilité de mises à jour continues et de corrections et d'améliorations illimitées.

Est actuellement en projet une version en castillan, en collaboration avec les Universités de Murcia, de Càceres et de Barcelone, dont sont actuellement disponibles les lettres B, R et quelques exemples. En outre, une convention de fraîche date avec l'Université de Grenoble-Stendhal nous autorise à promettre une version française, grâce, pour l'instant, à une bourse financée par la Région Rhône-Alpes.

\section{Exemples}

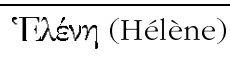

ELENA

Figlia di Zeus e di Leda, sorella di Castore e Polluce, moglie di Menelao (Hom. Il. passim)

Si tratta di un nome di difficile interpretazione, del quale Chantraine (DELG) ritiene sia inutile ricercare un'etimologia, perché probabilmente non greco.

Già Eschilo ( $A g$. 689-90) lo riteneva un nome parlante collegato con il tema $\leqq \lambda-$,

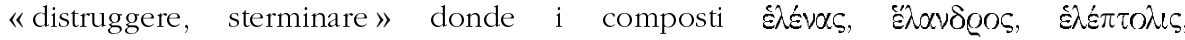
« distruggitrice di navi, sterminatrice di uomini e di città ».

Secondo Nilsson (Geschichte der griechischen Religion I, p. 315) si tratterebbe di un'antica dea della vegetazione minoica, da cui il nome di pianta घ̇ 2 Évov.

Carnoy (DEMGR.) ipotizza una derivazione dall'indoeuropeo *suel-, "brillare », trattandosi di una dea luminosa, sorella dei Dioscuri; anche von Kamptz (Homerische Personennamen, p. 136) è di questa opinione e in modo particolare ritiene che il nome derivi da §̇ $\lambda \dot{\alpha} \nu r_{1}$, « fiaccola ».

È attestato anche il maschile Eleno ("Thevoc), figlio di Priamo ed Ecuba e gemello di Cassandra (Hom. Il. 6, 76; 7, 44), come lei dotato di facoltà profetiche.

Wathelet (Dictionnaire des Troyens de l'Iliade, p. 509) ritiene improbabile che si tratti di una parola di origine straniera proprio per la presenza molto attestata del

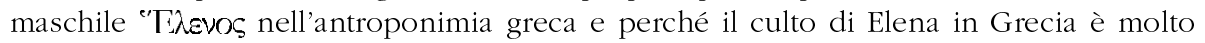
antico. 


\section{Eủgím (Europe)} EUROPA

Nome di diverse eroine, la piú celebre delle quali è la figlia di Agenore e Telefassa, amata da Zeus tramutatosi in un candido torello (Hom. Il. 14, 321 ss.).

Secondo Chantraine (DELG) l'etimologia è sconosciuta;

Frisk invece (Griech. Et. Wört., p. 593) ritiene che possa trattarsi di un nome pregreco: questa ipotesi, sostenuta anche da Sommer («Indog. Forsch. » 55, 1937, p. 185 n. 1), non sembra molto plausibile.

Berger (P.-W. s. v., VI col. 1287) ritiene che sia un composto di aủgúc, «ampio » $\mathrm{e}$

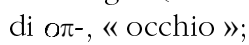

altri significati sono: « che risuona da lontano » (cr-, "voce ») e « oscuro », dati già dagli antichi.

Collegamenti sono stati operati anche col semitico (Grimme, «Glotta » 14, 1925, p. 17; cfr. la puntuale sintesi di Cássola, Studi Mazzarino, pp. 48-54), o addirittura con l'accadico erebu «tramonto », cioè « terra del tramonto, dell'occidente ».

Cássola propende con ragione per riconoscere un composto da eủgúc e - -uџ, «dall'ampio sguardo». Certo è che i greci dovevano intendere questo nome come analogo ai molti altri antroponimi femminili in $-\omega \downarrow$ probabile il significato: «dall'ampio volto».

Improponibili, per un nome femminile, le proposte di collegare il primo membro del composto a eủoúaıc, « caliginoso, muffito, oscuro ».

\section{7xy@é́s (Zagrèus) \\ ZAGREO}

Nome di un'antica divinità ctonia; figlio di Zeus e Persefone, identificato spesso con Dioniso.

Le ipotesi piú probabili e piú seguite sono due.

La prima, proposta da Chantraine (DELG), da B. Mader (in Snell, Lex. frühgr. Ep.) e da Perpillou (Les substantifs grecs en -suc, \389) è quella della derivazione dal nome

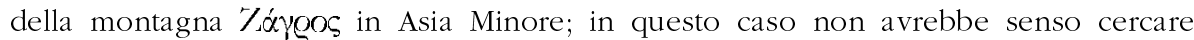
un'etimologia all'interno del greco.

La seconda, proposta da Frisk (Gr. Et. Wört.) e approvata anch'essa da B. Mader, è quella di un confronto con $\zeta \alpha_{\alpha} \gamma g \eta_{1}$, « trappola per animali », che si spiegherebbe con un

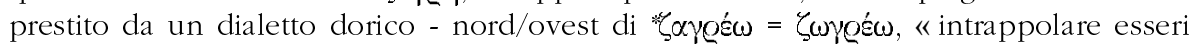
viventi », «catturare prede vive». Chantraine ritiene invece questo rapporto indimostrabile.

Carnoy $(D E M G R)$ propone che si tratti di un derivato dal pelasgico $\zeta_{\alpha}^{\alpha} \gamma \mathcal{C} \alpha$, derivante dall'indoeuropeo " $g h \mathrm{gh}$, ampliamento da $g h e$, «rimanere a bocca aperta », che si ritrova per esempio nell'antico islandese gj grar, «fessura di roccia »: sarebbe quindi avvenuta un'assibilazione della - $g$ -

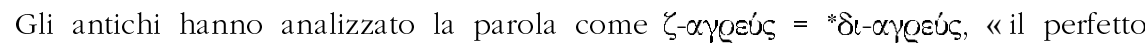
cacciatore », ipotesi accettata da Pape e Benseler (WGE): si tratta di un'etimologia popolare. 